\title{
PWE-058 ENTERIC DYSMOTILITY/ACQUIRED CHRONIC INTESTINAL PSEUDOOBSTRUCTION (ED/CIPO)- RELATIONSHIP BETWEEN SMALL INTESTINAL MANOMETRIC AND NEUROHISTOPATHOLOGICAL FINDINGS
}

doi:10.1136/gut.2011.239301.321

A T M D H Chowdhury, ${ }^{1,2, *}$ A Raimundo, ${ }^{1}$ A Darzi, ${ }^{1,2} \mathrm{~J}$ E Martin, ${ }^{3}$ D Silk1,2 ${ }^{1}$ Neurogastroenterology Unit, Princess Grace Hospital, London, UK; ${ }^{2}$ Department of Academic Surgery, Imperial College London, London, UK; ${ }^{3} T$ he Pathology Group, Barts and the Royal London School of Medicine, London, UK

Introduction Enteric dysmotility is an important clinical subgroup of the gastrointestinal neuromuscular disorders. ${ }^{1}$ Of a personal series of 130 patients recorded on our data base 89 have undergone both $24 \mathrm{~h}$ ambulatory studies of small intestinal motility and laparoscopic full thickness jejuna biopsies. The aim of the present study was to examine the relationship between the manometric and neurohistopathological findings.

Methods Prospective qualitative analysis of small intestinal motility tracings and abnormalities of 15 parameters of phasic activities of the intestinal interdigestive Migrating Motor Complex (IMMC) together with the occurrence of nocturnal abdominal pain during the study were documented. Neurohistopathological analysis of the full thickness biopsies was performed as previously described. ${ }^{2}$ 
Results All the small intestinal motility studies were abnormal 83 of 89 full thickness biopsies (93.3\%) were abnormal. $51(57.3 \%) \alpha$ actin epitope deficiency alone, $26(29.2 \%) \alpha$ actin epitope deficiency with other pathology, 6 (6.7\%) other pathology alone. Nocturnal abdominal pain was significantly related to the presence of vasculitis $(p<0.05)$ and smooth muscle atrophy $(p<0.05)$. Sustained uncoordinated phasic pressure activity and discrete cluster contractions were both related to myenteric plexus pathology $(\mathrm{p}<006, \mathrm{p}<0.04)$. Aberrant phase III $\mathrm{MMC}$ configuration was related to smooth muscle biopsies $(p<0.003)$ and smooth muscle atrophy $(p<0.05)$ as well as vasculitis $(\mathrm{p}<0.05)$.

A reduction in numbers of phase III MMC was related to vasculitis $(p<0.007)$ and smooth muscle atrophy $(p<0.007)$. Generalised low pressure activity was related to amyloid deposition $(p<0.002)$ and lack of fed pattern to the deposition of amphophilic bodies ( $p<0.03$ ).

Conclusion The present findings suggest that abnormal small intestinal manometry is a good indicator of the presence of underlying neuromuscular pathology. Patients suspected of having ED/CIPO with abnormal manometry should undergo laparoscopic full thickness biopsies.

Competing interests None.

\section{REFERENCES}

1. Knowles et al. Gut 2010;59:882-7.

2. Chowdhury et al. Gastroenterology 2010. 